\title{
Germanica
}

GERMANICA 7|1990

Grenze und Entgrenzung

\section{Karl Hillebrand Revolutionär, Grenzgänger, Europäer}

Wolfram Mauser

\section{CpenEdition}

1 Journals

Édition électronique

URL : http://journals.openedition.org/germanica/2485

DOI : 10.4000/germanica.2485

ISSN : 2107-0784

Éditeur

Université de Lille

Édition imprimée

Date de publication : 30 juin 1990

Pagination : 15-25

ISSN : 0984-2632

\section{Référence électronique}

Wolfram Mauser, « Karl Hillebrand Revolutionär, Grenzgänger, Europäer », Germanica [Online], 7 | 1990, Online erschienen am: 17 Juli 2014, abgerufen am 06 Oktober 2020. URL : http://

journals.openedition.org/germanica/2485; DOI : https://doi.org/10.4000/germanica.2485

Ce document a été généré automatiquement le 6 octobre 2020.

(c) Tous droits réservés 


\title{
Karl Hillebrand Revolutionär, Grenzgänger, Europäer
}

\author{
Wolfram Mauser
}

1 Vor einhundertvierzig Jahren wurde der Rhein, unweit von Straßburg, Zeuge dramatischen Geschehens. Unter dem Beschuß aufgescheuchter Wachsoldaten schwammen zwei junge Deutsche zum westlichen Ufer, um dort Sicherheit zu finden. Gewiß, über die Jahrhunderte war es immer wieder geschehen, daß Verfolgte jeweils auf der anderen Seite des Stromes Schutz suchten. Der vorliegende Fall verdient es, im Rahmen dieses Kolloquiums in Erinnerung gerufen zu werden. Denn der eine der Männer, die hier die Grenze überwanden, um sich der Vollstreckung des Todesurteils zu entziehen, wurde Jahre später zu einem der profiliertesten Europäer im Kultur- und Geistesleben des 19. Jahrhunderts: Karl Hillebrand, ein Gelehrter und Publizist hohen Ranges $^{1}$, über dessen Essays sich Friedrich Nietzsche freute, «als ob es Milch und Honig wäre. O Bücher, aus denen eine europäische Luft weht, und nicht der liebe nationale Stickstoff!».

2 Europäer zu sein, war in dem vertrackt nationalistischen Europa des 19. Jahrhunderts offenbar schon eine Kategorie der Anerkennung. Aber was war es, was damals Europäertum ausmachen konnte? Was bedeutete es, Europäer zu sein? Wie konnte man Europäer werden? Wie konnte Karl Hillebrand es werden? Enttäuschung und vielfache Desillusionierung hatten gewiß einen großen Anteil an seiner Abkehr von nationalem Dünkel. Doch nicht jeder politisch Enttäuschte wurde zum Europäer. Hillebrands geistige Entwicklung hing offenbar mit den Erfahrungen zusammen, die er in mehreren Ländern Europas machte; sie ließen ihn einen ganz eigenen Weg finden, als Autor und als politischen Denker. Die Anfänge dazu lagen hier am Oberrhein, im deutschfranzösischen Grenzgebiet, in der Regio.

3 Man schrieb den Oktober 1849. Die Revolution war überall in Europa geschlagen. Da entzündete sich an einem Soldatenkrawall in der Markgrafschaft Baden ein neuer Revolutionsherd. Noch einmal zogen junge Männer dorthin, wo sie glaubten, sich für Freiheit und Vaterland in die Bresche werfen zu können. Einer von ihnen war Karl Hillebrand, der Sohn Joseph Hillebrands, des angesehenen liberal-demokratischen 
Professors für Philosophie und Literatur in Heidelberg und Gießen, der sich auch als Abgeordneter zum Landtag in Hessen einen Namen machte.

Hillebrand, seit dem Frühjahr 1848 Student der Universität Gießen, war wie viele seiner Kommilitonen von den revolutionären Ideen der Zeit erfüllt. In einer Versammlung, in welcher der Vizekanzler der Universität mit Bierflaschen von der Tribüne gejagt wurde, jubelten ihm die Studenten wie einem Volkstribun zu. Als Starkenburger Corpsstudent mit der roten Mütze trieb es ihn dann - von diesem eher zweifelhaften Erfolg berauscht - überall dorthin, wo etwas los war: nach Frankfurt auf die Barrikaden, dann in die Pfalz und nach Baden. Dazwischen lagen Wochen, in denen er untertauchen mußtei, auch jenseits des Rheins soll er Zuflucht gesucht haben. 1849 schloß er sich dann dem bewaffneten Kampf um die deutsche Reichsverfassung in Baden an. Über seine Heldentaten weiß man wenig; gewiß war er in Gefechte verwickelt. Jedenfalls wurde er zusammen mit den Truppen Corvins gefangen, in die Festung Rastatt gebracht und zum Tode verurteilt. Täglich erwartete man seine Hinrichtung.

5 Was niemand für möglich gehalten hatte: $\mathrm{Ihm}$ und einem der Mithäftlinge gelang auf abenteuerliche Weise die Flucht aus den Kasematten von Rastatt. Sieben Stunden brauchten die beiden, bis sie alle Hindernisse überwunden hatten. Doch Wachen gab es auch draußen, und so half am Ende nichts als der Sprung in den kalten Rhein. Schwimmend und mit Hilfe eines Fischers gelangten sie auf die andere Seite des Flusses. Südlich von Beinheim gingen sie an Land. In einem langen unveröffentlichten Gedicht über die Flucht heißt es u.a.:

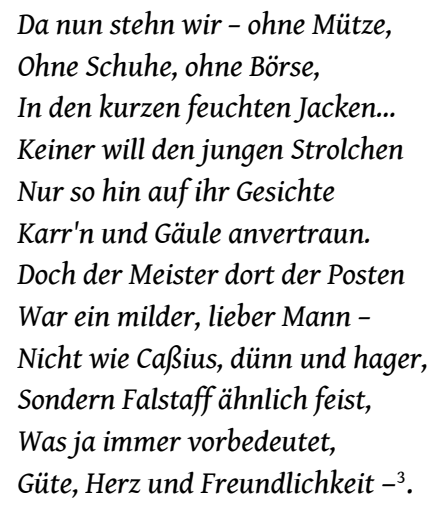

6 Unter solchen Umständen - aller Habe beraubt, aber freundlich aufgenommen begann die Karriere eines Mannes, der als europaweit angesehener Essayist und Publizist später - sozusagen - alle staatlichen Grenzen hinter sich lassen sollte. Er ging nach Straßburg und von dort nach Paris. Eine Zeitlang arbeitete er als Sekretär Heinrich Heines, der ihm aus seiner Matratzengruft den größten Teil des Romanzero diktierte. 1851 ließ er sich dann, über Vermittlung englischer Freunde, in Bordeaux nieder. Hier holte er den gesamten französischen Ausbildungsgang nach. Viele Jahre lang war ja an eine Rückkehr nach Deutschland nicht zu denken; erst 1858 wurde das Todesurteil aufgehoben; 1857 hatte er allerdings schon die französische Staatsbürgerschaft angenommen.

7 Allmählich gewann Hillebrand Distanz zu seinen «Jugendeseleien» ${ }^{4}$, wie er später das revolutionäre Gehabe nannte. Er erkannte, wie viel Dilettantismus mit dem Treiben der rebellischen Studenten verbunden war. Zunächst hatte ihn das Scheitern aller Bemühungen um Freiheit und Vaterland tief erschüttert, in der neuen Umwelt wurde $\mathrm{ihm}$ aber bewußt, wie kläglich es um die Verhältnisse in Deutschland eigentlich bestellt 
war, um die institutionalisierte Macht nicht weniger als um die Widersacher des Systems.

8 Hillebrands Neuanfang in Bordeaux erscheint im nachhinein wie der erste Schritt zu einem kulturpolitischen Programm. Er wechselte von Jura zur Philologie. Was ihn besonders anzog, waren die Renaissance und die Aufklärung. Sein Buch über Dino Compagni (1862) fand auch in Italien weithin Anerkennung. Seine Schrift Des conditions de la bonne comédie (1863) wurde von der Akademie in Bordeaux preisgekrönt und mit der ungewöhnlich hohen Summe von 500 Francs ausgezeichnet. Ehe Hillebrand Bordeaux 1863 verließ, hielt er eine Reihe von vierzehn öffentlichen Vorträgen, in denen er Goethes Werk in die großen europäischen Zusammenhänge stellte. Die Zeitung $\mathrm{La}$ Gironde hob den komparatistischen Aspekt der Arbeit des Vierunddreißigjährigen besonders hervor, und dies in einer Zeit, in der man überall und ganz selbstverständlich «National-Literaturen» schrieb: «... toute son œuvre est imprégnée d'une mentalité profondément "comparatiste"»

9 Die weitere Karriere ist schnell erzählt. Von Saint-Cyr ging Hillebrand an die Universität Douai bei Lille, er wohnte aber in Paris. Dort wurde er zu einem hoch aktiven und angesehenen Mitarbeiter der Revue des deux mondes, des Journal des débats, und der Revue moderne. Er schrieb vor allem über Gegenstände der deutschen, italienischen und englischen Literatur und zunehmend auch über Fragen des geistiggeselligen Lebens. Große Aufmerksamkeit fand eine Folge von drei umfangreichen Beiträgen über Die Berliner Gesellschaft von 1789-1815 in der Revue des deux mondes. 1865 reiste er als offizieller Vertreter der französischen Regierung zu den Dantefeiern nach Florenz und in den Jahren danach im Auftrag des Kultusministers Duruy nach Deutschland; als Ergebnis dieser Reisen legte er die Schrift De la réforme de l'enseignement supérieur vor. Nicht weniger Aufmerksamkeit als dieser Beitrag zur französischen Schulreform fand eine Folge von Essays im Journal de débats über La Prusse contemporaine et ses institutions, die dann auch in Buchform herauskam (1867).

10 Hillebrand war in das französische Kulturleben hineingewachsen wie wenige Deutsche vor ihm. So mußte ihn der Krieg von 1870 tief treffen. Mit aller Kraft hatte er sich der Verständigung zwischen Deutschen und Franzosen gewidmet. Die Ironie des Schicksals wollte es nun, daß er von einer aufgebrachten Volksmenge am Bahnhof in Lille, die sich wahllos gegen alles wandte, was mit Deutschland zu tun hatte, beinahe gelyncht wurde. So blieb ihm, ein zweites Mal, nichts anderes als die Flucht.

11 Er ging nach England und von dort im Auftrag der Times als Korrespondent nach Rom, um über den Einmarsch der italienischen Truppen in die Heilige Stadt zu berichten. Seine Korrespondenzen - lebendig, frisch und mitgeprägt vom Fieber der Ereignisse stellen noch heute eine lohnende Lektüre dar. Nach Abschluß der Mission ließ er sich in Florenz nieder, wo er 1884 starb. Die Wahl des Ortes war keineswegs zufällig. Florenz war für ihn die Wiege besten Europäertums. Persönliche Freiheit, Aufgeklärtheit und elitäre Geistigkeit wurden hier - nach seiner Ansicht - nicht nur gepflegt, sondern auch in Kunstwerke von höchster Vollendung umgesetzt.

Hatte sich für Hillebrand in Frankreich eine komparatistische Lehre und Publizistik wie von selbst ergeben, so machte er sie nun zu einem Programm. Alle, auch die ehrenvollsten Rufe auf Professorenstellen lehnte er ab. Mehr als zehn Jahre lang stellte er seine ganze Kraft in den Dienst der Vermittlung zwischen den europäischen Völkern. Zunächst erschien, sozusagen als Pendant zu La Prusse contemporaine, das Buch Frankreich und die Franzosen in der zweiten Hälfte des 19. Jahrhunderts. Damit war ein 
Muster gesetzt: Fortan schrieb er in jedem der vier großen europäischen Länder Deutschland, England, Frankreich, Italien - jeweils über das andere in der Absicht, die geistigen, literarischen und sozialen Zusammenhänge den Nachbarn zu erklären. Dabei erbrachte er nicht nur eine überragende darstellerische Leistung, seine Themen erforderten auch ein kaum vorstellbares Ausmaß an Lektüre, Nachforschungen und Gesprächen. Soweit seine Essays in deutscher Sprache erschienen, gehören sie zum Besten, was wir an kulturkritischer und biographischer Essayistik besitzen. Aber auch in den anderen Sprachen verfügte er über eine ungewöhnlich lebendige, frische und treffsichere Darstellungsweise.

Der Komparatist Hillebrand war auch einer der ersten Literatuxsoziologen. Gewiß, sein soziologischer Ansatz ist durch keine gesellschaftstheorie neueren Zuschnitts abgesichert, eher durch so etwas wie eine Geselligkeitstheorie, die ihre Leitvorstellungen dem geselligen Leben des 18. Jahrhunderts verdankte. Wir würden heute eher von einer Kultur Soziologie sprechen, die Montesquieu ebenso zu ihren Vätern zählte wie Johann Gottfried Herder. Nicht einer Theorie also verdankte Hillebrand die besondere Art seines Zugriffs, sondern den wechselvollen Erfahrungen seines Lebens. Er fühlte sich als Zeuge einer widersprüchlichen, aber bewegten Phase der europäischen Geschichte, und so stand für ihn außer Frage, daß der besondere Charakter einzelner Völker am besten über die Zeugenschaft einzelner Personen zu erfassen sei. Eine «curiosità sterminata», eine "grenzenlose Wißbegier», beseelte ihn, und sie war ihm auch die Gewähr für die Verläßlichkeit historischen Verstehens ${ }^{6}$.

Seine deutschsprachigen Essays erschienen übrigens in sieben Bänden Zeiten, Völker und Menschen, die eine Reihe von Auflagen erlebten und später in Auswahlausgaben immer wieder aufgelegt wurden. $\mathrm{Zu}$ den Spitzenleistungen literatursoziologischer Essayistik gehören Aufsätze wie Zur Entwicklungsgeschichte der abendländischen Weltanschauung, Zur Entwicklungsgeschichte der abendländischen Gesellschaft, Die Werther-Krankheit in Europa, Torquato Tasso, Defoe und Robinson Crusoe, Lorenzo di Medici, Montesquieu u.a.

15 Vergleichende Literaturund Kultursoziologie in vier europäischen Sprachen -: ist es dies, was ihn zum Europäer machte? Man hat es oft so gesehen. Der Punkt, den ich herausstellen möchte, ist aber ein anderer: In einem ganz spezifischen Sinne war es die Aporie der Grenze - zu verbinden, indem sie trennt -, die seinem Europäertum zugrundelag. Dazu eine kurze Vorüberlegung.

Wenn wir heute nach dem Zweiten Weltkrieg «Europa» sagen, denken wir an ein Europa, das zwischen den Großmächten seinen Platz definieren und sich behaupten muß. Ohne Zweifel stehen dabei wirtschaftliche Gesichtspunkte im Vordergrund. Die politischen Entscheidungen leiten sich vielfach davon ab, und die kulturelle Zusammenarbeit stellt oft nicht mehr als Begleitmusik dar. Und doch sehen wir in der Entwicklung, die wir erleben, wichtige Schritte in eine Zukunft, von der man annimmt, daß sie sich auf neu gewonnene Strukturen stützt.

Von frühesten Zeiten an war Europa, über die geographischen Gegebenheiten hinaus, nichts Festes und Konstantes, sondern eine immer wieder gefundene, aber auch immer wieder vergessene Klammer, die in bestimmten Situationen Antagonistisches, Widersprüchliches, ja Paradoxes auf spannungsvolle Weise zusammenhielt. Eine solche Gemeinsamkeit kam offenbar am ehesten dann zustande, wenn Gefahr drohte: beim Einbruch der Hunnen oder beim Vormarsch der Türken. Aber auch dann, wenn es galt, die Vormachtstellung einzelner abzuwehren: die des Hauses Habsburg, die Napoleons, die Preußens. Europa, eine Krisenideologie? Dynamisch vor allem dann, wenn es zu 
reagieren galt? Ein regulierender und konstruktiver Faktor vor allem in der Abwehr? Man wird dies nicht bestreiten können. Und doch ist dies nur eine Seite.

So etwas wie eine europäische Identität bildete sich erstmals im Wirkungsbereich der römischen Kirche aus. Die gemeinsame lateinische Sprache, das gemeinsame Ritual, weithin gemeinsame Kunstformen, aber auch große Gemeinschaftsunternehmungen wie die Kreuzzüge wirkten einheitsstiftend in einem Europa, das aus außerordentlich heterogenen Elementen bestand. Dieses Europakonzept zerfiel jedoch mit der konfessionellen Spaltung.

Was dann vor allem vom 18. Jahrhundert an - über die zentrifugalen Kräfte hinweg eine europäische Gemeinsamkeit begründete, war die wissenschaftlich-technischindustrielle Entwicklung. Eine europäische Zivilisation bildete sich heraus, zu deren konstitutiven Faktoren gehörten: kritische Vernunft, Naturbeherrschung, konsequente Organisation der menschlichen Arbeit, Mobilisierung von Energiequellen, Expansion und Maximierung. Und eng damit verbunden: Naturrechtsvorstellungen, Individualismus, Autonomie, Gleichheitsprinzip, Freiheitsideen. Unter solchen Voraussetzungen formierte sich das, was man später Kapitalismus nannte, verdichtete sich Macht in nationalen Staaten und Staatsapparaten, gelang es aber auch, Parlamentarismus, Demokratie und soziale Reformen durchzusetzen.

Vor diesem Hintergrund entfaltete Hillebrand die Idee eines Europa, zu der alle europäischen Völker und Staaten ihren gleichwertigen Beitrag geleistet haben und weiterhin leisten sollen. Dem Prinzip der volkhaft-nationalistischen Legitimation von Macht und Vorrang, aber auch dem technisch-industriell orientierten Zivilisationsoptimismus, der in Wahrheit neue Formen des Inhumanen produzierte, stellte er die Idee eines Europa entgegen, die das Verbindende der europäischen Völker und nicht das sie Trennende betont. Die Völker Europas bilden aus seiner Sicht eine große, vielfältige, aber doch in sich abgeschlossene Familie. Sie verwirklichen eine eigene, im ganzen gemeinsame Entelechie. In diesem Sinne verkörpern sie eine europäische Identität. Die Beiträge der einzelnen Völker zur europäische Gesamtkultur sah Hillebrand als Elemente eines - heute würden wir sagen - Interaktionsprozesses. Kulturelle Interaktion zwischen den Völkern sei der eigentliche Motor jeder Entwicklung, auch die Gewähr für politisch-wirtschaftlichen Fortschritt. Als historisch gewordene Einheiten hätten die Völker Europas in vielfacher Weise einander ergänzt. Indem sie wechselseitig aufeinander einwirkten, bezeugten sie nicht nur ihre Steigerungsfähigkeit, sie erfüllten auch die Idee einer höheren Einheit, einer übergreifenden Harmonie, eines Kultur-Ganzen, das seinen Sinn in sich trage. Kernstück von Hillebrands Bildungs-Europäertum war das humanistische Gymnasium vor allem deutscher (also Humboldtscher) Prägung; seine formende Kraft sei imstande, nicht nur die sozialen Spannungen, sondern auch nationale Vorurteile, ja chauvinistische Arroganz zu überwinden. In vielen Punkten berührten sich Hillebrands Ideen mit Nietzsches Kulturkritik. Was ihn vom Basler Philosophen aber unterschied, war sein Plädoyer für Toleranz und sein Respekt vor den jeweils anderen: «Die Denkfreiheit ist die moderne Kultur» ${ }^{7}$.

21 Bei aller Sympathie für Hillebrands Europakonzept, das sich auf Kultur, Bildung und Toleranz gründete, und für seine Europaperspektive, die auf Einheit, Ganzheit und Harmonie zielte, müssen wir erkennen, daß wir seine Ideen heute nicht einfach teilen oder übernehmen können. Und doch stellt sein Wirken eine Leistung von unbestreitbarer Aktualität dar. Einer Folie gleich läßt sich an seinem Beispiel das die 
Anschauungen Unterscheidende, das Überholte und das nach wie vor Gültige ausmachen.

Ich überspitze meine These: Was Hillebrand mit Verve und Bravour offensiv vertrat, war seiner Ideenlage nach Gedankengut des 19. Jahrhunderts; wie hätte es auch anders sein können. Was seinen Lebensweg, sein aktives Wirken und die Art seines Zugriffs im ganzen bestimmte, war indes - auch aus heutiger Sicht - bestes Europäertum. Damit meine ich das Folgende.

Das, worauf seine Anstrengungen des Ermitteins und Vermitteins zielten, war die Erkenntnis des Spezifischen im jeweils Anderen. Das Interesse am. Einzelnen und Besonderen bestimmte seinen Blick und sein Urteil. Als leitendes Prinzip diente ihm nicht die imaginierte Einheit, sondern die jeweils zu entdeckende Eigentümlichkeit, nicht das überwölbende Ganze, sondern das qualifizierend Unterscheidende, nicht die ersehnte Harmonie, sondern das Charakterisierende. Seine die Literatur, Gesellschaft und Kultur vergleichenden Essays lebten geradezu davon, daß er die Lebendigkeit, das Flair, den Glanz einer geistig-kulturellen Erscheinung wahrnahm und in einer sensibel differenzierenden Sprache festhielt. Was da zählte, war die Vielfalt, nicht das Einheitbildende, war das Sich-Unt er scheidende, nicht das EinanderEntsprechende, war das Verbindende, aber nicht in der Angleichung, sondern in der Differenz; und nicht um die Einschmelzung der charakteristischen Elemente sollte es gehen, sondern um deren Bewahrung 8 .

1881, von der Krankheit gezeichnet, notierte Hillebrand in Arcachon bei Bordeaux (mit einiger Erregung) im Tagebuch, daß sein Gesprächspartner, ein naturalisierter Franzose deutscher Herkunft, völlig die Fähigkeit verloren habe, «die Dinge von außen zu betrachten $»^{9}$. Der mehrfache Blick, das sei es, worauf es ankomme. Durch ihn werde es möglich, das Trennende, die Grenzen wahrzunehmen und sich zugleich im ständigen Überschreiten dieser Grenzen den Horizont des eigenen Weltverstehens zu erweitern. Das Andere ist dann nicht das Fremde oder Bedrohende, sondern die Herausforderung, sich auf die Besonderheit des Anderen und des Eigenen $\mathrm{zu}$ besinnen und es nebeneinander bestehen zu lassen. Erfahrung und Nachdenken lehrten ihn, daß es nicht auf Identität aus Übereinstimmung ankomme, sondern auf ein Miteinander aus Zuwendung, Wissen, Respekt und Solidarität. Dieses Modell einer übernationalen Verständigung war im 19. Jahrhundert in keinem Lande mehrheitsfähig, seine konstruktive Kraft entfaltet es erst heute. Das in der Welt von damals «Unzeitgemäße» (Nietzsche) an Hillebrand erweist sich mehr als hundert Jahre später als das politisch und menschlich Gebotene.

Mag sein, daß der «Falstaff» unter den Zöllnern an der französischen Seite des Rheins mit «Güte, Herz und Freundlichkeit», wie es hieß - ohne es zu wissen dazu beitrug, daß aus einem frierend nassen revolutionär ein Mann wurde, in dessen Bewußtsein das Phänomen Grenze den Charakter des Ein- und des Ausgrenzens verlor; an die Stelle nationalen Denkens trat mit zunehmender Vertrautheit des Fremden die Fähigkeit, das eigene in einem neuen Erfahrungszusammenhang zu sehen und beides, das Fremde und das Eigene, in seiner jeweiligen Besonderheit nicht nur als kulturelle Leistung zu verstehen und $\mathrm{zu}$ akzeptieren, sondern sich auch emotional damit verbunden $\mathrm{zu}$ fühlen. Viele Zeugnisse belegen, daß Hillebrand in diesem Sinne Europäer war, daß für ihn Grenzen nicht ein Element des Trennens, sondern des Verbindens darstellten. 


\section{NOTES}

1. - Zu Karl Hillebrand: Wolfram Mauser: Karl Hillebrand. Leben, Werk, Wirkung. Dornbirn 1960 (Gesetz und Wandel 1). Dort und im folgenden weitere Literatur. Darüber hinaus: F.C. Greeley Stahl: Karl Hillebrand et la France (ungedruckte Dissertation Paris 1972 bei Charles Dédéyan). Rudolf Vierhaus: Zeitgeschichte und Zeitkritik im essayistischen Werk Karl Hillebrands. In : Historische Zeitschrift 221, 1975, S. 304-325. Elsbeth Wolffheim: Von Heine zu Nietzsche. Zum 100. Todestag von Karl Hillebrand. In: Castrum Peregrini 164/65,1984, S. 59-78. Jean Nurdin : L'idée d'Europe de la pensée allemande à l'époque bismarckienne. Metz 1977 ; darin : Karl Hillebrand et la crise de la culture occidentale S. 589-633. Wichtig: Karl Hillebrand. Mostra di documenti a cura di Lucia Borghese, Florenz 1984 (Ausstellungskatalog), und Lucia Borghese (Hg.): Karl Hillebrand. Eretico d'Europa. Atti del seminario (1-2 novembre 1984). Florenz 1986, 316 S. (Gabinetto Scientifico Letterario G.P. Vieusseux. Studi 3).

2. - Friedrich Nietzsche und Karl Hillebrand. Unveröffentlichte Briefe, in : Süddeutsche Monatshefte 6/2, 1909, S. 129-142, Brief von Mitte April 1878. Jetzt : Nietzsches Briefwechsel Kritische Gesamt-ausgabe. Hg. von Giorgio Colli und Mazzino Montinari. Bd. II, 5. Berlin 1980, S. 318.

3. - Das Original der Handschrift Hillebrands ging im Zweiten Weltkrieg verloren, eine Kopie blieb jedoch erhalten.

4. - Otto Hartwig: Karl Hillebrand. In: O.H.: Aus dem Leben eines deutschen Bibliothekars. Marburg 1906, S. 140. (Ursprünglich Nachruf in der Beilage zur Münchner Allgemeinen Zeitung 1885, Nr. 98, 99 und 100).

5. - La Gironde, 13. August 1863. Zitiert nach: Greeley Stahl: Karl Hillebrand à Bordeaux. Documents retrouvés. In : Revue historique de Bordeaux et du département de la Gironde. 1878/79, S. 99-117, Zitat S. 115.

6. - Heinrich Homberger: Karl Hillebrand. In : H.H. : Ausgewählte Schriften. Essays und Fragmente. München 1928, S. 65-107, Zitat S. 78. (Ursprünglich Nachruf in der Nation 29. November 1884, mehrfach z.T. verändert nachgedruckt).

7. - Karl Hillebrand: Zeitgenossen und Zeitgenössisches. Berlin 1882, S. 331 (Zeiten, Völker und Menschen, Bd 6).

8. - Die Aktualität von Hillebrands Europa-Konzept zeigt sich nicht nur deutlich im Vergleich zur Position etwa des Staatslexikons von 1861 (dazu Wolfram Mauser, in: Borghese, Hillebrand, 1986, S. 15; siehe Anmerkung 1), sondern besonders auch zu früheren Schriften, so zu J. Weitzel: Europa in seinem gegenwärtigen Zustande. Wiesbaden 1824, der im einzelnen zeigt, daß sich in der eigenen «höchst denkwürdigen Epoche» die «natürlichen» nationalen Grenzen weitgehend aufgelöst hätten. Erstmals träten zwei «Parteien» in Erscheinung, «die sich bei aller Verschiedenheit in Sprache, Religion und Regierungsform, durch die entferntesten Länder befreundet die Hände reichen, und, bei aller Gleichheit in Sprache, Religion und Regierungsform, sich scheiden, wo nicht hassen und verfolgen. Die Natur hat, durch den Verkehr der Völker und durch die Entwickelung der geistigen Anlagen des Menschen, sehr viel von ihrem Einflüsse auf denselben verloren, und die freie Überlegung und Wahl ist an die Stelle der physischen Nöthigung getreten. Die Nationen sind weniger durch klimatische und geographische Einwirkungen als durch die der Gesinnungen und Gefühle verbunden und geschieden; und auf diesem Wege haben die Staaten die so veränderte Gestalt gewonnen, von der die Rede ist. Sie sind nicht mehr, wie früher, in sich abgeschlossene Ganze, sondern enthalten Theile, die mit denen anderer Staaten in näherer Verwandschaft stehen, als mit den Gliedern des eignen Staates. Die zwei Parteien, in die sich die Welt theilt, kann man, da die Sache einen Namen haben soll, die revolutionäre und die gegenrevolutionäre nennen. Sie stehen, wie die beiden Pole, sich einander 
unverträglich und unversöhnlich gegenüber, haben entgegengesetzte Zwecke, die sie indessen durch dieselben Mittel zu erreichen suchen. Hier heißt Recht, Vernunft und Gesetz, was dort für Unrecht, Aberwitz und Willkühr gehalten wird» (S. 13-14). Nicht diese Art von politischgeistiggesellschaftlicher Spaltung und Auflösung alter politischer und gesellschaftlicher Ordnungen ist es, worin Hillebrand grenzüberschreitende Perspektiven seiner Zeit sah, sondern eine diese Positionen überschreitende Vorstellung von Einheit in der Vielfalt. Sie war wohl nur denkbar aus der Enttäuschung, in die die revolutionär-liberale Bewegung ebenso geführt hatte wie die konservativ-restaurative.

9. - Handschriftliches Tagebuch aus Anlaß eines Kuraufenthaltes in Arcachon bei Bordeaux 1881. 